\title{
LANDAU DAMPING OF THE WEAK HEAD-TAIL INSTABILITY AT TEVATRON*
}

\author{
P.M. Ivanov\#, Y. Alexahin, J. Annala, V.A. Lebedev, V. Shiltsev, FNAL, Batavia, IL 60510, USA.
}

\section{Abstract}

Two-step improvement of transverse beam stability has been done at the Tevatron. First, shielding of the laminated Lambertson magnets significantly reduced the transverse impedance. Second, Landau damping induced by the octupoles allowed machine operation with zero chromaticity at injection. That has resulted in a dynamic aperture increase and weakening of long-range beambeam effects due to smaller chromatic tune modulation. At collisions, the coherent tunes and incoherent tune continuum are separated due to the head-on collisions. That leads to Landau damping loss at small chromaticities. Predictions of the analytical Landau damping model are compared with experiments.

\section{INTRODUCTION}

The transverse head-tail (HT) instability has been a long-standing problem in the Tevatron operation. It was predicted in Ref. [1] that the major source of the impedance responsible for its excitation comes from the Lambertson injection magnets. Four 0.4 mm-thick shielding liners with a total length of about 12 meters have been inserted inside these magnets to reduce significantly the impedance and to mitigate the instability but the problem was not fully resolved. The lattice chromaticities required to stabilize the beam in multibunch mode ( $\sim 5$ at injection and $\sim 18$ at collisions) were still too large. They cause the strong chromatic modulation of betatron tunes and, consequently, the degradation of the beam lifetime. As a result, the antiproton beam lifetime at injection is completely determined by the long-range beam-beam effects. Recently, to suppress the instability at small chromaticities we tried another approach. It is based on Landau damping from the octupole-generated tune spread and allows weakening of the long-range beam-beam effects for both proton and antiproton beams at injection energy due to machine operation at zero chromaticities. Unfortunately, we could not apply this approach at collisions where the head-on beam-beam interactions cause separation of the coherent tunes and incoherent continuum leading to a loss of Landau damping. The existing machine octupoles were rearranged into four new families to control the betatron tune spread and the differential chromaticity between the proton and antiproton helical orbits [2].

\section{BEAM STABILITY AT INJECTION}

The head-tail stability regions in the chromaticity planes have been measured with a single proton bunch with the regular intensity before (curves 1-2 in Fig.1) and after installation of the liners (curve 3). Curves 1 (central orbit) and 2 (proton injection orbit) correspond to the different orbit positions inside Lambertson magnets (see Fig.2). The instability thresholds were determined by observing fast growth of Schottky power near the threshold. After the liner installation the HT stability region does not depend on the beam positions inside the Lambertson magnets. With the 5 times lowered transverse impedance on the proton injection orbit, Landau damping from the synchrotron frequency spread stabilizes all HT modes at positive chromaticities as shown in Fig. 1. At negative chromaticities, the beam stability is limited by excitation of the lowest order, $m_{s}=0$, head-tail mode with the maximum growth rate achieved at $\xi_{x} \approx \xi_{y} \approx-3$ units.

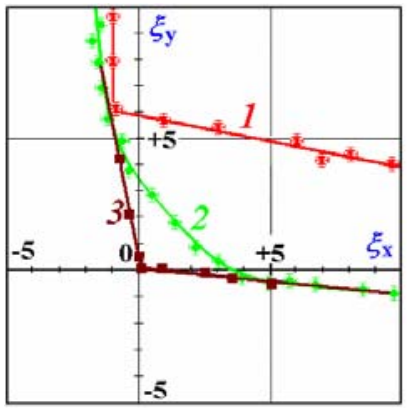

Fig.1. Head-tail stability bounds Fig in the chromaticity plane.

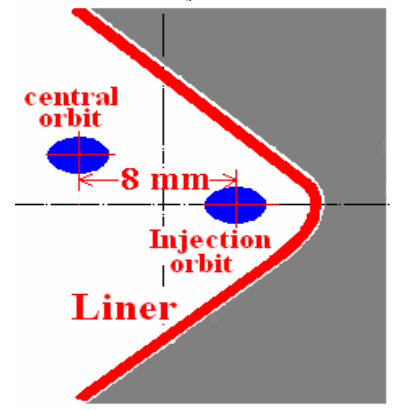

Fig.2. Proton beam positions at the injection magnet notch.
Two different polarity sets for the octupole families $\mathrm{OF}$ and OD have been examined with a single proton bunch [2]. Both of them stabilize all HT modes at chromaticities $\xi_{x}, \xi_{y} \geq-4$. The set with alternate polarities has demonstrated larger transverse dynamic aperture and has been put into Tevatron operation. The beam stability is worse if all 36 bunches (Np 2.6.1011 per bunch) are injected. In this case, the transverse coupled bunch instability at the lowest negative betatron frequency is developing if the horizontal chromaticity of $\xi_{\mathrm{x}} \leq 2$. Experimentally we observed the excitation of two different modes in the chromaticity range of $0<\xi_{x}<2$ [3]. Even switching-on the octupoles does not give rise to stabilizing if $\xi_{\mathrm{x}}<2$. This is connected with an "incorrect" sign of the cubic non-linearity in the horizontal plane that has been set to attain a maximum dynamic aperture. Increasing the OF-octupole strength will result in widening of the tune spread but Landau damping cannot occur because the incoherent continuum maximum will move away from the coherent tune lines.

\section{LANDAU DAMPING MODEL}

In this section, Landau damping model is considered in the framework of the coherent oscillation theory stated in [4]. The frequency spectrum of the coherent oscillations

\footnotetext{
* Work supported by the U.S. Department of Energy under contract No. DE-AC02-76CH03000....

\#pmivanov@fnal.gov
} 
can be described by the dispersive integral equation obtained from the linearized Vlasov equation:

$$
\varepsilon\left(v_{\mathbf{c o h}}\right)=1-\frac{\Omega_{\mathbf{m}}}{A_{\mathbf{m}} \omega_{o}} \int_{0}^{\infty} d J_{y} d J_{x} d J_{s} \frac{J_{y}^{m_{y} \mid} J_{x}^{\left|m_{x}\right|}\left(\mathbf{m}_{\perp} \partial F_{o} / \partial J_{\perp}\right)}{\mathbf{v}_{\mathbf{c o h}}-\mathbf{v}_{\mathbf{m}}\left(J_{y}, J_{x}, J_{s}\right)}=0
$$

Here $v_{\mathbf{c o h}}=v_{y}-v_{y}(0)$ is the detuning of a mode, $\Omega_{\mathbf{m}}$ is the complex coherent frequency shift for a given mode in the absence of tune spread; $\boldsymbol{v}_{\mathbf{m}}=\mathbf{m} \cdot v_{y, x, s} ; \mathbf{m}=\left(m_{x}, m_{y}, m_{s}\right)$ is the coherent oscillation multipole number, and $A_{\mathbf{m}}$ is a normalizing factor.

Below we consider the stability condition of the most unstable mode, $\mathbf{m}=(0,1,0)$. Adding the effect of space charge not accounted for Eq. (1) we obtain:

$$
\varepsilon\left(v_{\mathbf{c o h}}\right)=1-\left[\frac{\Omega_{m}}{\omega_{O}}+\Delta q_{y}\right] \int_{-\infty}^{\infty} d\left(\Delta v_{y}\right) \frac{G_{y}\left(\Delta v_{y}\right)}{v_{\mathbf{c o h}}-\Delta v_{y}+\Delta q_{y}+i \varepsilon}=0
$$

where $\Delta q_{y}$ is the peak value of the linear space charge tune shift, the tune spectral function $\mathrm{G}_{y}\left(\Delta v_{y}\right)$ is given by:

$$
G_{y}\left(\Delta v_{y}\right)=\frac{N_{b}}{\varepsilon_{x} \varepsilon_{y} \varepsilon_{s}} \int J_{y} \frac{\partial F_{o}\left(J_{y}, J_{x}, J_{s}\right)}{\partial J_{y}} \delta\left[\Delta v_{y}-\Delta v_{y}\left(J_{y}, J_{x}, J_{S}\right)\right] d J_{x} d J_{y} d J_{s} .
$$

Here $\varepsilon_{x, y, z}$ are the beam emittances. The tune spread introduced by the beam space charge is small relative to the octupole-generated tune spread. Therefore only the linear part of the tune shift contribution is left in Eq. (2). The space charge shifts the tune spectral function by $\Delta q_{y}$ so that $G_{y}\left(\Delta v_{y}\right) \rightarrow G_{y}\left(\Delta v_{y}-\Delta q_{y}\right)$. Hence, it contributes to the coherent tune shift as capacitive impedance, $\Omega_{m} / \omega_{0} \rightarrow$ $\Omega_{m} / \omega_{0}+\Delta q_{y}$. This approach was verified by formal derivation not considered here due to the lack of space.

The charge distribution function $F_{o}\left(J_{x}, J_{y}, J_{S}\right)$ can be presented as a product of the Gaussian transverse and parabolic longitudinal distributions

$$
\begin{gathered}
F_{o}\left(J_{y}, J_{x}, J_{s}\right)=f_{o}\left(J_{y}, J_{x}\right) \cdot \rho_{o}\left(J_{s}\right), \\
f_{o}\left(J_{y}, J_{x}\right)=\exp \left(-J_{y} / \varepsilon_{y}-J_{x} / \varepsilon_{x}\right) / \varepsilon_{x} \varepsilon_{y}, \\
\rho_{o}\left(J_{s}\right)=2\left(1-J_{s} / 3 \varepsilon_{s}\right) / 3 \varepsilon_{s} .
\end{gathered}
$$

All degrees of freedom contribute into incoherent tune spread due to the octupole cubic non-linearity and quadratic chromaticity. For on-momentum particles $\left(J_{S}=0\right)$ Eq. (3) can be reduced to the following form:

$g_{y}\left(\Delta v_{y}\right)=\frac{N_{b}}{\varepsilon_{x} \varepsilon_{y}} \int d J_{x} d J_{y} J_{y} \frac{\partial f_{o}\left(J_{y}, J_{x}\right)}{\partial J_{y}} \delta\left[\Delta v_{y}-\Delta v_{y}\left(J_{y}, J_{x}\right)\right]$,

where the amplitude-dependent detuning $\Delta v_{y}\left(J_{y}, J_{x}\right)$ is expressed in terms of the cubic non-linearity coefficients,

$\Delta v_{y}\left(J_{y}, J_{x}\right)=v_{y}\left(J_{y}, J_{x}\right)-v_{y}(0)=\alpha_{y y} J_{y}-\alpha_{x y} J_{x}$.

As a result of integration in Eq. (7) the spectral function $g_{y}\left(\Delta v_{y}\right)$ is of the form of

$$
\begin{array}{r}
g_{y}\left(\Delta v_{y}\right)=\frac{2 N_{b}}{w}\left(\frac{\Delta v_{y}}{w_{y y}}+\frac{w_{x y}}{w}\right) \exp \left(\frac{\Delta v_{y}}{w_{y y}}\right) \cdot \Phi\left(\Delta v_{y}\right)+ \\
+\frac{2 N_{b} w_{x y}}{w^{2}} \exp \left(\frac{\Delta v_{y}}{w_{x y}}\right) \cdot \Phi\left(-\Delta v_{y}\right),
\end{array}
$$

where $\Phi(x)=\{1$ for $\mathrm{x} \geq 0,0$ for $\mathrm{x}<0\}$ is the Heaviside step function, and $w=\alpha_{y y} \varepsilon_{y}+\alpha_{x y} \varepsilon_{x}=w_{y y}+w_{x y}$ is the effective width of the betatron tune spread. Taking into account the momentum spread, the tune spectral function $G_{y}\left(\Delta v_{y}\right)$ of Eq. (3) is rewritten as,

$$
G_{y}\left(\Delta v_{y}\right)=\int_{0}^{J s \max } g_{y}\left[\Delta v_{y}-\overline{\Delta v_{y}\left(J_{s}\right)}\right] \rho_{o}\left(J_{s}\right) d J_{s}
$$

A chromatic detuning for off-momentum particles in Eq. (10) is caused by the quadratic lattice chromaticity and the modulation of space charge tune shift by synchrotron oscillations. In accordance with the analytic formalism developed in [2] it is given by:

$$
\overline{\Delta v_{y}\left(J_{s}\right)}=\left(6 \xi_{2 y} \cdot \sigma_{\Delta p / p}^{2}+\Delta q_{y}\right) \cdot J_{s} / 6 \varepsilon_{s}-\Delta q_{y} .
$$

Integration in Eq. (10) with this chromatic detuning yields a piecewise continuous function. The expression is too long and it is not included in this paper. The plot of the spectral function $\mathrm{G}_{y}\left(\Delta v_{y}\right)$ for the alternate polarity octupole set is presented in Fig. 3. The vertical tune distribution function $F_{y}\left(\Delta v_{y}\right)$ (dotted line 1 in Fig.3) is calculated in accordance with Ref. [2] and the same beam parameters.

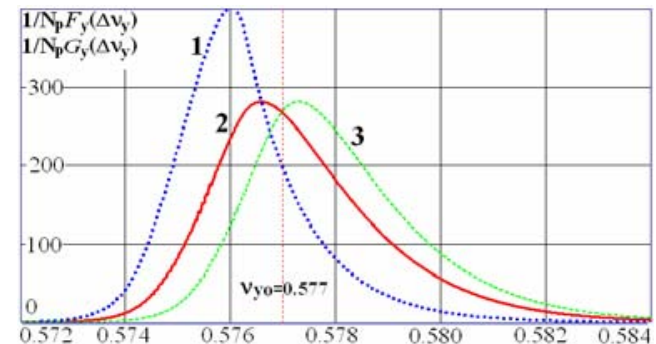

Fig.3.The vertical betatron tune distribution $F_{y}\left(\Delta v_{y}\right)$ (curve-1) and the corresponding spectral function $\mathrm{G}_{y}\left(\Delta v_{y}\right)$ (curve-2) with octupole family settings of $\boldsymbol{I}_{\boldsymbol{O D}}=7.0 \mathrm{~A}$ and $\boldsymbol{I}_{\boldsymbol{O}}=-6.0 \mathrm{~A}$. Curve 3 is the same as the curve 2 but the space charge effect is absent.

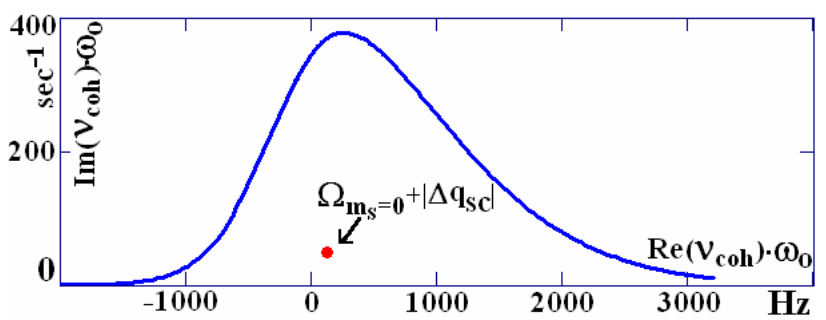

Fig. 4. Stability diagram for the mode $\mathbf{m}=(0,1,0)$ and beam parameters of Figure 3.

We follow the standard method to build the stability diagram. Solving Eq. (2) relative to $\Omega_{\mathrm{m}}$ and computing the integral numerically for real values of $v_{c o h}$ with the spectral function $\mathrm{G}_{y}\left(\Delta v_{y}\right)$ presented in Figure 3, we obtain the dependence $\Omega_{\mathrm{m}}\left(v_{c o h}\right)$ that inscribes the stability region 
in the plane of complex coherent frequency shift. Figure 4 presents the results of the computations. The point below the curve corresponds to the Tevatron complex coherent frequency shift for the $(0,1,0)$ mode that is equal to $\Omega_{\mathrm{m}} \approx$ $-90+\mathrm{i} 50 \mathrm{~s}^{-1}$. It was extrapolated from the measurements of the instability growth rate and the tune dependence on the beam current performed before the liner installation for the bunch intensity of $N_{p} \approx 2.7 \cdot 10^{11}$ and chromaticity $\xi_{y} \approx-3$.

\section{BEAM STABILITY AT COLLISIONS}

The preliminary beam studies show that a single proton bunch is unstable at the collision energy if both lattice chromaticities are decreased below 5-6 units. It points out that the synchrotron and betatron tune spreads are insufficient to prevent the instability if the octupoles are turned off. For the Tevatron the major contribution to transverse impedance comes from the wall resistivity. That result in that $R e \Omega_{m} \propto 1 / \mathrm{E}^{3 / 4}$, where we also took into account that the RF voltage stays constant during Tevatron operation. The synchrotron tune spread decreases with acceleration as $\Delta \omega_{s} \propto 1 / E$ (bunch shortening is taken into consideration). That yields the energy scaling law for the instability, $\Delta \omega_{s} / R e \Omega_{m} \propto 1 / E^{1 / 4}$, resulting in $\Delta \omega_{s} / \operatorname{Re}\left(\Omega_{m}\right)$ to be decreased by a factor of 1.6 at the collision energy. On the other hand, the contribution of lattice quadratic chromaticities to the betatron line widths becomes almost neglible because it scales with energy as $\left(\sigma_{\Delta p / p}\right)^{2} \propto 1 / E^{3 / 2}$. In multi-bunch mode the beam is stabilized if the chromaticities are increased above 15-20. Although the nature of this instability has not been clearly identified, an estimate shows that Landau damping from the octupole-generated betatron tune spread could stabilize the beam at zero chromaticities if there are no head-on beam-beam collisions.

\section{Betatron tune spread due to head-on collisions}

In the case of a Gaussian round antiproton beam the dependence of proton tune on betatron amplitudes due to head-on collisions can be expressed by the following approximate equation:

$$
\Delta v_{x, y}^{b b}\left(J_{x}, J_{y}\right) \approx \frac{4 n_{c} \xi_{p} \varepsilon_{0 a}}{2 J_{x, y}+J_{y, x}+4 \varepsilon_{0 a}} .
$$

Here $\xi_{p}=r_{p} N_{a p b} / 4 \pi \gamma \varepsilon_{0 a}$ is the beam-beam parameter, and $n_{c}=2$ is the number of head-on collisions per turn. The betatron tune distribution function due to the head-on collision for the Gaussian proton beam is defined as

$\left.\rho\left(v_{x, y}^{b b}\right)=\frac{1}{\varepsilon_{p x} \varepsilon_{p y}} \int_{00}^{\infty} \int_{0}^{\infty} d J_{x} d J_{y} e^{-\left(\frac{J_{x}}{\varepsilon_{p x}}+J_{y}\right.}\right) \delta\left[v_{x, y}-\Delta v_{x, y}^{b b}\left(J_{x}, J_{y}\right)\right]$

Integration in Eq. (13) yields:

$$
\begin{gathered}
\rho\left(\Delta v_{x, y}^{b b}\right)=\frac{4 \xi_{p} n_{c} \varepsilon_{0 a}}{\Delta v_{x, y}^{2}\left(2 \varepsilon_{p x, p y}-\varepsilon_{p y, p x}\right)} \exp \left(-\frac{2 \varepsilon_{0 a}\left(\xi_{p} n_{c}-\Delta v_{x, y}\right)}{\Delta v_{x, y} \varepsilon_{p x}}\right) \\
x\left(1-\exp \left(-\frac{2 \varepsilon_{0 a}\left(2 \varepsilon_{p x, p y}-\varepsilon_{p y, p x}\right)\left(\xi_{p} n_{c}-\Delta v_{x, y}\right)}{\Delta v_{x, y} \varepsilon_{p x} \varepsilon_{p y}}\right)\right)
\end{gathered}
$$

The tune distribution function (Eq. (14)) is determined in the tune range of $\left[v_{o}, 2 \xi_{p}\right]$ and it is normalized as $\int \rho\left(\Delta v_{x, y}^{b b}\right) d v_{x, y}=1$. Figures 5-6 show the tune distributions calculated using Eq. (14) at the beginning and end of a typical HEP store. It has been seen that the coherent tunes for some HT and coupled bunch modes are out of the incoherent continuum generated by head-on collisions. This results in loss of Landau damping at small chromaticities. At the beginning of HEP store, switchingon the octupoles even with maximum integral strengths do not significantly change the tune spread (solid line in Fig. 5). The tune distribution function with octupoles is calculated by applying Monte-Carlo method.

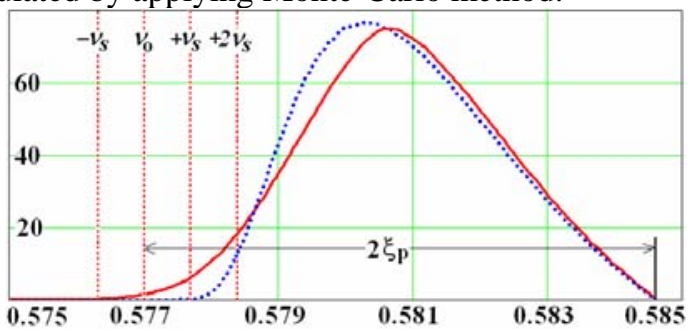

Fig.5. Calculated betatron tune spread due to head-on collisions (blue dot line) for the proton beam at the start of a HEP store. Introduction of the octupoles with maximal integral strengths does not change the tune spread significantly (solid red line).

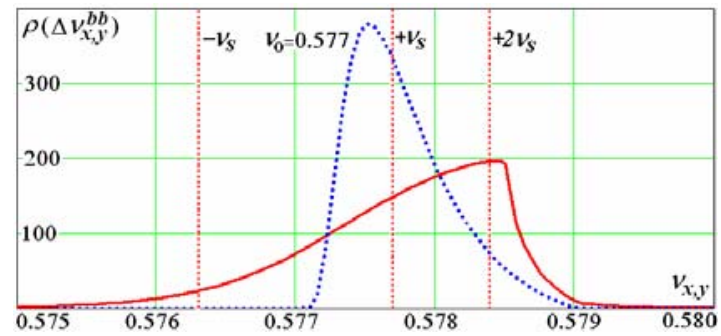

Fig. 6. Calculated betatron tune spread due to head-on collisions (dotted line) for the proton beam at the end of a HEP store. At small chromaticities Landau damping is lost only for rigid bunch modes $\left(m_{s}=0\right)$ of the coupled bunch instability. The solid line shows the tune spread with the octupoles turned on.

\section{CONCLUSIONS}

The described above actions allowed us to operate Tevatron at zero chromaticities at low energy. That significantly improved the beam lifetime at the injection but more work is required to achieve this at collisions. We plan further optimization of the octupole families and improvements of the transverse damper to operate with zero chromaticity at collisions. The developed theoretical approach has been useful in understanding the instability and aiming the effort to suppress it in conditions of limited study time of the operating collider. 


\section{REFERENCES}

[1] P.M.Ivanov et al., "Head-Tail Instability at Tevatron" Proc. of PAC 2003, p.3062

[2] P.M. Ivanov et al., Paper MPPP042, PAC 2005, these Proceedings, 2005.

[3] V. Lebedev, A. Burov, Proceedings of HB-2004 Workshop, Bensheim, Germany, 2004.

[4] N. Dikansky, D. Pestrikov, "The Physics of Intense Beams and Storage Rings”, AIP Press, NY, 1994.

[5] J. Gareyte, J.P. Koutchouk and F. Ruggiero, LHC Project Report 91, Geneva, 1997. 